\title{
Ventilation-perfusion (V/Q) lung scintigraphy: a long journey to a renewed position of prominence in diagnosing pulmonary embolism
}

\author{
Raphaella da Silva $\cdot$ Muhammad Shah • \\ Leonard M. Freeman
}

Received: 29 April 2014/ Accepted: 16 August 2014/Published online: 21 October 2014

(C) Italian Association of Nuclear Medicine and Molecular Imaging 2014

\begin{abstract}
The ventilation-perfusion (V/Q) lung scan has traveled a long, circuitous path since its introduction for the diagnosis of pulmonary embolism (PE) in the 1960s. Its initial credibility was damaged following the publication of the PIOPED study in 1990 and the emergence of computed tomographic pulmonary angiography (CTPA) in the mid-1990s. A considerable number of advances in both methodology and image interpretation have helped to restore the credibility of V/Q. There has also been considerable concern over the large radiation burden associated with CTPA. The issue of overdiagnosis and overtreatment of small PEs has also received a lot of attention. Although CTPA diagnoses more of these smaller PEs than V/Q, follow-up "outcomes" data show that they do not require treatment. The false-negative rates for both procedures are approximately $1 \%$ and not significantly different from one another. Finally, a change from traditional probability interpretations to a simpler and similarly accurate trinary system has made V/Q scintigraphy interpretations easier to understand for the referring clinician.
\end{abstract}

Keywords Pulmonary embolism - Ventilation-perfusion (V/Q) scintigraphy $\cdot$ Computed tomographic pulmonary angiography (CTPA)

\section{Introduction}

The ventilation-perfusion radionuclide lung study (V/Q) for pulmonary embolism (PE) has had a controversial

R. da Silva $\cdot$ M. Shah $\cdot$ L. M. Freeman $(\bowtie)$

Division of Nuclear Medicine, Department of Radiology,

Montefiore Medical Center, Albert Einstein College of

Medicine, 111 East 210th Street, Bronx, New York 10467, USA

e-mail: 1freeman@montefiore.org history since its introduction almost five decades ago. Disagreements over and changes to the interpretive criteria have been among the main reasons for this controversy. Computed tomographic pulmonary angiography (CTPA), introduced in the mid-1990's, was embraced by diagnostic radiologists, and then by clinicians, as a superior diagnostic tool for diagnosing PE [1]. In general, diagnostic radiologists are more comfortable with an anatomic imaging study, like CT, than with a functional imaging study, such as V/Q imaging. The shortcomings of the Prospective Investigation of Pulmonary Embolism Diagnosis (PIOPED), published in JAMA in 1990 [2], fueled the controversy over V/Q imaging and, in many people's minds, made CTPA a welcome option.

Recent concerns about both overdiagnosis and overtreatment of small PEs as well as the extremely high radiation burden associated with CTPA, together with major advances in the methodology and interpretation of V/Q scintigraphy have re-awakened interest in this nuclear medicine study. We now have data to prove that V/Q scintigraphy is equal to CTPA in diagnosing clinically significant $\mathrm{PE}$ and carries considerably less radiation risk for the patient.

\section{The Biello classification-1979 [3]}

In 1979, Dr. Dan Biello and his colleagues at Washington University in St. Louis provided us with a very useful interpretive scheme based on probability assessment. Two or more large V/Q mismatches $(>75 \%$ of the anatomic bronchopulmonary segment) were classed as high probability ( $>85 \%$ likelihood of PE), while two or more small mismatches $(<25 \%$ of the anatomic segment) were classed as low probability $(<10 \%$ likelihood of PE). Single, moderate-sized mismatches (25-75\% of segment) were 
defined intermediate. This classification and correlation were based on a retrospective review of V/Q exams and conventional catheter pulmonary angiography in 146 patients.

Patients unable to undergo a ventilation study have instead been investigated through comparison of perfusiononly scans and chest X-rays, correlating the size of $\mathrm{Q}$ defects with radiographic infiltrates. This perfusion-only methodology was validated and endorsed in the Prospective Investigative Study of Acute Pulmonary Embolism (PISA-PED) study [4].

It is interesting that both co-guest editors of this pulmonary issue of clinical and translational imaging were participants in PISA-PED publications.

It should be noted, as well, that another interpretive scheme using probability estimates was proposed by Dr. Barbara McNeil [5], but it did not achieve the popularity of the Biello classification.

\section{The PIOPED study [2]}

In the mid-1980s, a group of six institutions performed this prospective study comparing V/Q and pulmonary angiography in 755 patients. The specificity of both high probability (97\%) and normal/near normal studies was good, but low probability and intermediate interpretations showed considerable inter-observer variability: 70 and $75 \%$, respectively. Most importantly, there was an unacceptable rate $(44 \%)$ of intermediate interpretations.

\section{Problems with PIOPED}

The PIOPED study had several shortcomings:

1. $68 \%$ of the studied population were in-patients. This was one of the main reasons for the high (44\%) number of intermediate interpretations. These patients are more likely to have significant underlying cardiopulmonary problems and abnormal chest X-rays creating "triple matches" and thus resulting in useless, intermediate interpretations. In the later PIOPED II study evaluating CTPA, only $11 \%$ of the studied subjects were in-patients [6].

2. The low probability interpretation corresponded to a $20 \%$ likelihood of PE rather than the $10 \%$ threshold applied by Biello et al.

This is not acceptable to clinicians having to decide whether to institute anticoagulant therapy. The latter is associated with an $8 \%$ complication rate. Thus, Biello's $10 \%$ low probability interpretation is acceptable for assessing benefit-to-risk ratio whereas $20 \%$ is not. Subsequently, the revised PIOPED criteria included a "very low probability" category associated with a $<10 \%$ likelihood of PE [7, 8].

3. Single segmental mismatch (SSM).

The PIOPED investigators unfortunately categorized this feature as low probability which represented an incorrect change from Biello's classification of SSM as intermediate. The PIOPED results showed that $36 \%$ of patients with SSM had PE on angiography. SMM was thus re-categorized as intermediate in the Revised PIOPED report published in 1993 [8].

\section{The good things about PIOPED}

Since the study was performed in the mid to late 1980 s, it was one of the first investigations to have a computer database. This allowed several retrospective analyses of the data which greatly refined our interpretive skills [9]. The great importance of correlating the scintigraphic results with clinical data such as pre-test probability (Wells score) and the presence or absence of underlying cardiopulmonary disease became evident [10, 11]. For example, an $87 \%$ positive predictive value (PPV) of a V/Q interpretation of high probability was found to rise to $96 \%$ when a high pre-test probability was also taken into account. Similarly, the $14 \%$ PPV of a low probability interpretation fell to $4 \%$ in patients with a low pre-test probability.

\section{The modified PIOPED (1993)}

Gottschalk et al. [8] undertook a retrospective study to try and correct some of the deficiencies apparent in the 1990 PIOPED study. The salient changes were:

1. Re-categorization of SSM, back into Biello's intermediate probability category.

2. Creation of a "very low" probability category which was associated with a $<10 \%$ likelihood of PE. The different findings placed in the very low category included such things as non-segmental perfusion abnormalities, stripe sign [12], triple matched defects in the upper and mid-lung fields [13], and several other findings summarized in a review article by Freeman et al. [9].

\section{The rise of CTPA and the PIOPED II study}

The popularity of CTPA grew in the mid to late 1990s. This was attributable, in part, to the post-PIOPED decline of interest in V/Q imaging. Additionally, it is a given fact 
that the great majority of diagnostic radiologists are more comfortable interpreting anatomic images of the pulmonary vasculature rather than functional V/Q images. This preference extends to clinicians whose consultant radiologists recommend CTPA over V/Q [14]. Articles appearing in emergency medicine literature describe this preferred algorithm of using CTPA as the primary imaging tool because of its greater accuracy [15].

To better establish the role of CTPA in diagnosing PE, a group of eight centers formed a PIOPED II investigative team whose findings were published in 2006 [6]. A total of 824 patients were entered in this team's study in which the composite reference standard for the diagnosis of $\mathrm{PE}$ included: V/Q scintigraphy, lower extremity Doppler sonography for deep venous thrombosis (DVT), Wells score and pulmonary digital subtraction angiography when available. Interestingly, V/Q scintigraphy represented a significant component of what constituted the "truth" in PE diagnosis.

Of the 824 patients, $87(10.6 \%)$ were excluded from the analysis because of inconclusive results. CT venography of the lower extremities was also a part of this study. The reported sensitivity and specificity for PE diagnosis was 83 and $96 \%$, respectively. However, if the inconclusive cases were included, these values dropped to $78 \%$ sensitivity and $90 \%$ specificity.

The great power of correlating the results with pre-test probability was again demonstrated. The PPV for PE was $86 \%$, but when correlated with a low Wells Score, it plunged to $58 \%$. Interestingly, this turned out to be similar to what was seen in PIOPED I where the combination of a high probability V/Q and a low Wells score was associated with a PPV of $56 \%$ [2]. In its very final sentence, the PIOPED II report states that "additional testing is necessary when clinical probability is inconsistent with the imaging results".

\section{Enhanced interpretation of V/Q scintigraphy [9]}

Pre-existence of cardiopulmonary disease

As mentioned previously, the presence of significant underlying cardiopulmonary disease makes interpretation more difficult. Currently, we require a chest radiograph prior to commencing a V/Q study. The great majority of these are negative. If significant abnormalities exist, a CTPA is recommended. Of course, renal insufficiency and contrast media allergies are contraindications to CTPA and in such circumstances V/Q may be necessary.
Correlation with objective clinical assessment (Wells score)

As also indicated previously, PIOPED proved this to be a powerful tool. Of interest, in this regard, is the current status of the SSM. The revised PIOPED had, as mentioned, put this feature back into Biello's intermediate probability classification. Stein et al. [10] looked at the SSM in a retrospective review of PIOPED I. They showed that a SSM in a patient with no significant cardiopulmonary disease, i.e., negative chest X-ray, had a PPV of $86 \%$, making it high probability. Furthermore, when it was associated with a high pre-test probability, the PPV rose to $100 \%$. Currently, we classify a well-defined SSM in association with a negative chest X-ray as positive for PE.

\section{Ancillary scintigraphic findings}

A large number of ancillary findings have greatly strengthened our interpretive skills. These are described in detail in a review by Freeman et al. [9]. A brief description of several of the most useful signs is given here:

1. The "stripe" sign

This was first described by Sostman et al. in the early 1980s [12].

Subsequently, it was retrospectively reviewed and validated as it applied to the PIOPED I study [16]. It consists of a consistently visualized stripe of normally perfused lung interposed between a perfusion defect and adjacent pleural surfaces. When present in the PIOPED study, it was associated with a very strong negative predictive value (NPV) of $93 \%$.

2. Fissure sign

This consists of a linear band of decreased perfusion corresponding to either the major or minor lung fissures. Although originally described as a possible $\mathrm{PE}$, experience has shown that it is much more likely to be associated with pleural effusions or chronic parenchymal disease [17].

3. Pleural effusions

PEs are most often associated with small rather than large effusions.

Effusions occupying $1 / 3$ or more of the hemi-thorax with associated matched defects and no other areas of mismatch are not likely to represent PE [18].

4. Triple matched defects

Both Biello and the PIOPED I investigators categorized all V/Q matches associated with infiltrates or effusions (triple matches) as intermediate. Worsley et al. [13], in their retrospective review, showed that triple matches in the upper and mid-lung fields were 
associated with PE in $11 \%$ and $12 \%$ of cases, respectively. They could therefore be classified as low probability. Triple matches in the lower lung fields remained classifiable as intermediate probability since they were associated with a $33 \%$ incidence of PE.

5. "Reverse mismatch" defects

This term refers to studies where the ventilation defect is bigger than the perfusion defect. This finding is also a good negative predictor of PE [19].

6. Comparison of perfusion-only study and radiographic density

This approach is adopted by proponents of the PISAPED classification and is also applicable to situations in which a patient cannot perform a ventilation study. Defects larger than infiltrates are very suspicious for PE whereas defects smaller than infiltrates are low probability and those equal in size are intermediate. This was recognized by Biello in his original probability assessment-based interpretive scheme [3]. PISA-PED proponents, in particular, rely on the wedge shape of a perfusion defect [4].

Other signs such as the segmental contour pattern [20], pulmonary infarct sign [21], cephalization of flow, together with other normal variants, are described in the review article by Freeman et al. [9].

\section{Radiologists and clinicians are more comfortable with CTPA}

As indicated previously, the great majority of diagnostic radiologists are more comfortable with CTPA because it defines morphology rather than requiring them to assess the functional aspects highlighted by a V/Q study.

Unfortunately, most major US institutions do not adequately train their residents in V/Q interpretation. This philosophy was passed on to a second generation of radiologists, i.e., those who trained in the 1990s and are now teaching the current generation of residents. In addition, they justify their preferential use of CTPA because of its ability to detect other anatomic abnormalities, e.g., dissecting aneurysms, pneumonias, etc., that may present with similar symptomatology to PE. This justification has recently been seriously questioned [22].

Most significantly and unfortunately, clinicians in the US have been forced into practicing defensive medicine. The problem of escalating lawsuits is an ever-increasing concern among clinicians, particularly in the Emergency Department setting. The ready availability of CTPA has been detrimental to complete clinical evaluation. In most medical centers in the US, minimal symptomatology such as any dyspnea or apparently non-cardiac chest pain, mandates an immediate referral for CTPA, which is available around the clock. Conventional logic would indicate that a low pre-test likelihood of PE (low Wells or Geneva score) associated with a negative D-dimer assay should rule out any likelihood of PE and allow patient discharge. Interesting proof of this observation was provided in a recent article from the University of Arizona where a clinical low-risk assessment was associated with a $0.9 \%$ positivity on CTPA [23]. When coupled with a negative D-dimer, this fell to zero. A good reflection of these times is that D-dimer assays were performed in only $5 \%$ of the patients investigated in this study [23]. In addition to D-dimer levels, Doppler ultrasound leg studies should be utilized.

\section{Radiation exposure}

With the advent of multi-detector scanners, CTPA has become the de facto gold standard for imaging PE [24]. Several articles have appeared cautioning physicians about the high patient radiation exposure from CTPA [25-27]. In addition, recent articles in lay publications have cautioned the public. One, written by two California physicians and proclaiming "We are giving ourselves cancer", appeared in the New York Times [28]. The whole-body effective radiation dose from CTPA is approximately five times greater than that from V/Q imaging. Most significantly, the dose to the female breast is 20-40 times greater [29]. Parker and colleagues [30] demonstrated that $60 \%$ of patients evaluated with CTPA are women, of whom $26.7 \%$ are under the age of 40 . Breast tissue is certainly more glandular and radiosensitive in young women. This large breast radiation dose, which amounts to $>20$ rad, greatly exceeds the guideline of several radiologic organizations, including the American College of Radiology.

Dose rates for CTPA are typically in the same range as conventional chest CT, i.e., 2-4 rad (20-40 mGy) [30]. This compares with an effective radiation dose equivalent of $0.06-0.25 \mathrm{rad}(0.6-2.5 \mathrm{mGy})$ for a two-view chest radiograph and an average glandular breast dose of $0.3 \mathrm{rad}$ (300 $\mathrm{m}$ rad or $3 \mathrm{mGy}$ ) for a standard two-view screening mammogram [31]. Few physicians are aware that conventional diagnostic chest $\mathrm{CT}$ imparts a radiation dose of 2-5 rad (20-50 mGy) to the breasts of an average-sized woman. This dose is roughly equivalent to 10-25 two-view mammograms (a lifetime of mammograms) and up to as many as 100-400 chest radiographs [31, 32]. The extremely high radiation exposure associated with chest CT angiography has also been associated with increased lifetime risk of breast cancer [26]. 


\section{The pregnant patient with suspected $\mathrm{PE}$}

Articles in the obstetric literature have favored the use of CTPA over V/Q because of a reported lower radiation dose to the fetus. This argument is based on the fact that the abdomen can be lead-shielded during the CTPA exam. Ridiculously, though, it totally ignores the enormous radiation to the mother's breast. In fact, the pregnant woman's breasts are even more vascular and glandular than those of a non-gravid woman. Pahade et al. [29] estimated the radiation exposure to the gravid breast to vary between 1,000 and 2,000 mrad as compared to $22-28 \mathrm{mrad}$ from a V/Q scan. The fetal radiation dose was estimated to be slightly higher with V/Q (10-80 mrad) than with CTPA (1-66 mrad).

For the past 25-30 years, we, at New York's Montefiore Medical Center, have used low-dose $(1 \mathrm{mCi})$ perfusiononly studies with great success. A recent unpublished review of 324 patients revealed negative findings in $87.3 \%$, positive findings in $2.6 \%$ and intermediate findings in $10 \%$.

The intermediate results on these perfusion-only exams were most often seen in asthmatic patients where subsequent V/Q studies after bronchodilator therapy revealed normal results. A recent joint recommendation of the American Society of Thoracic Radiology and the American Thoracic Society recommends that Doppler ultrasound of the legs should be the initial exam in pregnant women suspected of having PE [33]. If the result is positive, anticoagulant therapy should be started; if it is negative, a chest radiograph should be performed. If the chest radiograph is negative, a V/Q scintigraphy should be performed, but if the chest radiograph is positive, CTPA should be performed. This algorithm has also been endorsed by the American College of Obstetrics and Gynecology.

The successful use of perfusion-only scintigraphy combined with a chest radiograph was described in the well-known PISA-PED study [4]. The authors performed a comparative trial of the modified PIOPED II and the PISAPED criteria. The sensitivity and specificity were statistically comparable (84.9 and $92.7 \%$, respectively, for modified PIOPED II and 80.5 and $96.6 \%$, respectively, for PISAPED). Of interest was the fact that the modified PIOPED II interpretations showed significantly more nondiagnostic readings $(20.6 \%)$ than did the PISAPED interpretations $(0 \%)$. Comparing the modified PIOPED perfusion, PISA-PED perfusion and CTPA, the PPVs were $72.4,84.7$ and $85.7 \%$, respectively, and the NPVs were $96.5,95.5$ and $94.8 \%$, respectively. These data support the position of the PISA-PED authors, namely that in the appropriate hands and with appropriate training, perfusiononly scans interpreted in association with a chest radiograph may suffice. Some, including these authors, argue that the addition of the ventilation study allows physicians to be more comfortable in interpreting these studies.

\section{The Montefiore/Einstein approach to studying patients with suspected PE}

In December 2006 and January 2007, three educational seminars were held between our Emergency Department attending, resident staff, the Director of Nuclear Medicine, the Chief of Radiology and the Section Chief of Cardiothoracic Radiology [34]. Information from the literature concerning radiation exposure and the relative accuracy of V/Q scanning and CTPA was discussed at these sessions.

The Emergency Department clinicians were given the following recommendations: a stable patient with a clinical suspicion of PE should initially be imaged with chest radiography. The rare unstable patient should have immediate CTPA regardless of chest X-ray findings. If the chest radiographic findings in stable patients are normal and further imaging for suspected $\mathrm{PE}$ is deemed appropriate on the basis of clinical assessment, a V/Q scan should be requested. If, on the other hand, the chest radiograph shows a significant pleural or parenchymal abnormality, the recommendation is to perform CTPA. If either examination proves equivocal or the imaging results are discordant with the clinical impression (objective clinical assessment), the Emergency Department staff should request the alternative test in addition. This algorithm was provided to the Emergency Department staff as a handout. All agreed that reducing patient radiation exposure would improve the care of appropriate stable patients despite the inconvenience of a slight delay in patient disposition. The algorithm was reinforced by a telephone call consultation and reminder from a radiologist or nuclear medicine physician every time CTPA was requested in an Emergency Department patient with a normal chest radiograph. If the differential diagnosis included aortic dissection, a CT was performed. In accordance with our usual clinical care, V/Q scanning was performed in patients with contraindications to CTPA (contrast media allergy or renal insufficiency) regardless of the findings on chest radiography. The algorithm was well accepted, but if it was not used for an individual patient, a dialog with the Emergency Department was initiated by the imaging services to discuss the specific details. In each case, the final decision on the appropriate imaging technique for an individual patient was left to the clinician caring for the patient in the Emergency Department. All V/Q studies were performed as an eight-view planar exam. SPECT V/Q, which is used in many institutions particularly outside of the US, was not part of the algorithm and has not been used. The efficacy of SPECT V/Q will be discussed later in this review. After successful 
Table 1 Three-month follow-up on negative CTPA studies and V/Q studies interpreted as normal, very low or low probability (or PEnegative), from 2009 to the present

\begin{tabular}{lll}
\hline & CTPA & V/Q \\
\hline \# of cases & 1799 & 3039 \\
False negatives & $16(0.9 \%)$ & $37(1.2 \%)$ \\
& $10 / 16$ PE 6/16 DVT & $14 / 37$ PE 23/37 DVT \\
FN for PE alone & $10 / 1799=0.6 \%$ & $14 / 3039=0.5 \%$ \\
\hline
\end{tabular}

CTPA computed tomographic pulmonary angiography, $V / Q$ ventilation/perfusion study, $P E$ pulmonary embolism, $D V T$ deep venous thrombosis

implementation of the protocol, the number of CTPA examinations performed decreased from 1,234 in 2006 to 920 in 2007, and the number of V/Q scans increased from 745 in 2006 to 1,216 in 2007 . The mean effective radiation dose to the patient was reduced by $20 \%$, from $8.0 \mathrm{mSv}$ in 2006 to $6.4 \mathrm{mSv}$ in $2007(p<0.0001)$. The patients who underwent CTPA and V/Q scanning in 2006 were of similar age. The patients who underwent V/Q scanning in 2007 were significantly younger. There was no significant difference in the false-negative (FN) rate (range 0.8-1.2\%) between CTPA and V/Q scanning in 2006 and 2007 [34].

The above-described algorithm has been utilized for the past $7 \frac{1}{2}$ years up to the time of this writing. The practice patterns of our physicians changed in response to this educational intervention, resulting in a reduction in radiation exposure to Emergency Department patients with suspected $\mathrm{PE}$ without compromising patient safety. Using this protocol, we have now performed more than 10,000 V/Q studies with continued success. The FN rates for both V/Q and CT are comparable at approximately $1 \%$ (Table 1 ). This is quite similar to what emerged from Anderson et al's Multi-institutional Prospective Study in Canada [35].

\section{The issue of overdiagnosis and overtreatment: the significance of small PE}

In a classic 2005 editorial in Radiology, Dr Larry Goodman elegantly discussed the significance of small PE [36]. He pointed out that there is good evidence that we all likely pass small PEs on a daily basis. These are lysed by fibrinolytic activity in the pulmonary arterial vasculature, which thereby protects the systemic circulation. He concludes that there are three indications for anticoagulating patients with small, peripheral PE. These are:

1. Co-existent DVT.

2. Significant underlying cardiopulmonary disease.

3. Chronic PE because of a concern regarding the development of pulmonary hypertension.
Filling defects in sub-segmental arteries as small as 2-3 mm in diameter can be detected in $15 \%$ of high-resolution CTPA [37]. However, direct comparison of CTPA to V/Q studies reveals that only $1 \%$ of V/Q scans interpreted as "high probability" correspond to an isolated subsegmental PE [38] compared with $15 \%$ of positive CTPA studies [37].

Incidental detection of small PE in studies performed for other indications occurred in $16 \%$ of mechanically ventilated patients [39], in $17 \%$ of patients over the age of 80 [40], and in $20 \%$ of trauma patients [41]. In addition, $50-60 \%$ of patients in a consecutive autopsy series were found to have an unsuspected PE when the pulmonary arteries were carefully dissected [42].

It is quite clear that CTPA detects more PE than V/Q scintigraphy does. In Anderson's prospective study, the positivity of PE was $17.2 \%$ for CTPA and $11.2 \%$ for V/Q [35]. Despite this, the FN rate based on three-month follow-up was $0.4 \%$ for CTPA and $0.7 \%$ for V/Q. Anderson's study also included CT study of the leg veins. If DVT was included in the follow-up analysis, the FN rate for CT remained the same, while V/Q rose to a still statistically insignificant $1 \%$. One of the most striking features of the study by Anderson et al. was their criteria for a technically adequate diagnostic CTPA. All that required was adequate visualization of the main and lobar pulmonary vessels. It was not necessary to visualize the segmental vessels. Despite this relatively limited vessel opacification, the FN rate was $0.4 \%$. This constitutes strong support for the concept of overdiagnosis of PE.

Strong epidemiological support for the overdiagnosis of PE is provided by two studies promoted by Montefiore's Chief of Cardiothoracic Radiology, Dr. Linda Haramati. In the first study the hospitalization records of 12 million New York State patients showed that the advent of CTPA in the mid-1990s had the effect of doubling the number of admissions for PE [43]. Despite this fact, the mortality rate remained very low and did not change between the pre- and post-CTPA eras. Another more recent study dealing with dedicated Montefiore patients showed similar results [44]. Therefore, the ability of CTPA to detect twice as many PEs had an insignificant impact on patient's survival. It is, therefore, reasonable to conclude that the anticoagulant treatment received following the enhanced CTPA detection of PE was not needed.

Traditionally, CTPA has been shown to be equivalent to V/Q scanning in three-month outcome studies, but it detects more clinically insignificant pulmonary emboli. Isolated subsegmental pulmonary emboli may account for overdiagnosis, but it must be asked whether the short-term likelihood of recurrent thromboembolism is lower than the risk of adverse events with anticoagulation in patients at high risk of hemorrhage. Donato and colleagues studied three-month 
clinical outcomes of a cohort of patients diagnosed with isolated subsegmental pulmonary emboli [45]. Review of 10,453 consecutive CTPA radiology reports over a 74-month period since the implementation of multidetector CT pulmonary angiography identified a cohort of 93 patients, from a single institution, found to have acute pulmonary embolism isolated to subsegmental pulmonary arteries without other evidence of DVT. The study measured three-month clinical outcomes (anticoagulation use, recurrence, death, hemorrhage) determined by review of records and telephone interviews with physicians. Seventy-one patients (76\%) were treated with anticoagulation and/or inferior vena cava filter, while 22 (24\%) were observed without therapy. One patient (1/93) who was treated with anticoagulants and a vena cava filter had a recurrent subsegmental pulmonary embolus. No patients died of PE. There were eight hemorrhages; including five $(5.3 \%)$ major hemorrhages without any hemorrhage-related mortality. The study also provided substantial evidence that small, subsegmental emboli may not need to be found.

At Montefiore, our algorithm with its preferential use of V/Q scintigraphy has been in place since January 2007. We have been able to obtain a minimum follow-up of 3 months on 3,039 V/Q studies and 1799 CTPA exams interpreted as normal, very low or low probability. More recently (see below), we combined these into a "PE negative" category using a trinary interpretive scheme.

The positivity of CTPA is approximately $14 \%$ while that of V/Q has varied between 6 and $9 \%$. These numbers are relatively similar to those reported by Anderson et al. [35]. As in their study, our FN rates for these two techniques have been found to be statistically similar. The follow-up looked at the subsequent development of either DVT or PE. Table 1 presents the results for both DVT and $\mathrm{PE}$, as well as for PE alone.

The increasing awareness of the issue of overdiagnosis and overtreatment has supported the use of the less sensitive V/Q study. The identical follow-up FN rates of CTPA and V/Q support this use of an outcome-based rather than an accuracybased methodology (Table 1). It has been suggested that anticoagulation can probably be withheld in patients with isolated subsegmental PE who are stable and have adequate cardiopulmonary reserve [46]. An ongoing prospective trial randomizing patients with small PEs between treatment and nontreatment should provide some definitive answers and, hopefully, support for not treating these patients [47].

\section{The role of single-photon emission computed tomography (SPECT) V/Q}

SPECT V/Q imaging has become the standard of care in most institutions outside the United States. A full issue of
Seminars in Nuclear Medicine, guest edited by Australia's Dale Bailey and Paul Roach, clearly makes the case for the improved accuracy of this methodology [48]. More recently, Marika Bajc and her colleagues wrote a superb editorial in the European Journal of Nuclear Medicine and Molecular Imaging extolling the virtues of SPECT V/Q [49]. There is unanimity amongst these authors and others [50] that the sensitivity of V/Q SPECT is superior to that of planar V/Q. The European Association of Nuclear Medicine's guidelines clearly express their preference for $\mathrm{V} / \mathrm{Q}$ SPECT based upon its greater sensitivity [51].

Our preference for planar rather than the more sensitive SPECT V/Q relates again to the overdiagnosis issue. The FN rate of SPECT V/Q is $1.5 \%$ [52], which is essentially the same as that of planar V/Q or CTPA (Table 1). Another point favoring planar $\mathrm{V} / \mathrm{Q}$ in the United States is the unavailability of Technegas ${ }^{\circledR}$ (Cylomedia Corp., Sydney Australia), which is universally recognized as the best ventilation agent. Ongoing clinical trials in the US will, hopefully, lead to FDA approval in the not too distant future. The current method used for ${ }^{99 \mathrm{~m}}$ Tc-DTPA aerosol studies at our Medical Center employs the "Swirler ${ }^{\circledR}$ ", (Amici Corp., Spring City, Pennsylvania) which has provided us with very good ventilation studies. The similarity between FN rates for planar and SPECT V/Q supports the fact that the additional emboli detected on the SPECT study are small peripheral lesions that do not need to be treated as long as there are no associated DVTs or underlying cardiopulmonary problems.

There is another important practical aspect supporting $\mathrm{V} / \mathrm{Q}$, this time of a geographical nature. Unfortunately, the constant concerns over medico-legal issues, as mentioned, force physicians in the US to practice defensive medicine. In most instances, any patient with a diagnosis of PE, regardless of size, will be anticoagulated. As previously pointed out, most small PEs do not require treatment. We know this because the FN rate is the same for CTPA, planar and SPECT V/Q. Most other countries do not share this US-based need to treat almost all PEs, regardless of size.

Two years ago, the senior author (LMF) had the opportunity to attend a pulmonary conference in Sydney, Australia, where the case was described of a young woman shown to have a small, peripheral PE on a SPECT V/Q exam. The pulmonologist managing the patient accepted the interpretation, but decided not to anticoagulate the patient since no other associated factors such as DVTs or underlying cardiopulmonary disease was present. Unfortunately, as indicated above, this is not the practice in the US where defensive medicine is practiced. Therefore, the fact that less sensitive planar V/Q does not pick up the very peripheral, clinically insignificant PEs works to our advantage, as well as that of the patient [34, 44]. 


\section{Chinese multicenter comparison of CTPA and V/Q}

A relatively recent prospective comparison of CTPA and V/Q was performed in China. Of interest is that both CTPA and $\mathrm{V} / \mathrm{Q}$ were performed in all 544 patients enrolled in the study [53]. Despite including patients with high, intermediate and low Wells scores, the investigators ended up with a high positive yield (59\%) compared with the PE prevalence rates of 33 and $19 \%$ reported, respectively, in PIOPED I [2] and PIOPED II [6]. The authors attributed this to their medical centers having a high-risk patient population.

Another important factor to be considered in China relates to the fact that patients must initially pay for their diagnostic studies out of their own pocket, and subsequently seek reimbursement from government insurance [personal communication]. As a result, they are more ready to question their doctors who, therefore, must be more judicious in ordering imaging studies. Pre-test probability is an important factor in aiding their decision on whether or not to request a study. This approach is much more restrictive than in the US and is probably an important factor in their high positive rate of $59 \%$ as compared to the $14 \%$ for CTPA and $9 \%$ for V/Q reported by Anderson et al. [35].

Strong follow-up evidence has thus far supported the practice of considering "outcomes" rather than "accuracy" in the management of PE. The results of the previously mentioned ongoing randomized multi-institutional study involving the withholding of treatment in patients with subsegmental PE should finally help to resolve the ongoing issue of how to manage small PEs [47].

\section{The language of lung scan interpretation}

The probability interpretations initially proposed by Biello and maintained through the two PIOPED eras have never been fully understood by referring physicians [54] or standardized satisfactorily by physicians interpreting the V/Q study [55]. This holds particularly true for the "low probability" interpretation which has varied between $\mathrm{Bi}$ ello's original $10 \%$ likelihood of PE up to $40 \%$. To address this issue, we implemented a trinary scheme of interpretation in 2009 where normal, very low probability and low probability were combined into a single category called "no evidence of PE". This has been received very favorably by our clinicians.

The safety and accuracy of the trinary system was retrospectively evaluated by Glaser et al. [56]. As before, the chest radiograph was utilized to determine the patient's candidacy for V/Q or CTPA. The great majority ( $>90 \%$ ) of patients have a negative or minimally abnormal radiograph and proceed to $\mathrm{V} / \mathrm{Q}$, whereas those with infiltrates or pleural effusions are triaged to CTPA provided it is not contraindicated because of renal insufficiency or contrast media allergy. Patients with negative radiographs are considered not to have any significant underlying cardiopulmonary disease. Consistent with the work of Stein and Gottschalk [10,11], this allows us to upgrade a welldefined SSM into the high probability category with a PPV of $86 \%$. Additionally, when associated with a high pre-test likelihood of disease, this value is upgraded to $100 \%$.

Our retrospective review of the effectiveness of the trinary interpretive scheme included 654 patients in a sixmonth period compared to 778 patients studied in the previous 6 months using the older "probability" scheme. The results were statistically similar with a FN rate of $1.14 \%(8 / 701)$ for the older, probability-based interpretation group and $1.5 \%(9 / 585)$ for the trinary interpretation group $(p=0.63)$. Of note was a significant difference in the "PE present" group which corresponded to $8.4 \%$ of the sample as compared to $4.9 \%$ in the prior "high probability" category. We attributed this to the more aggressive criteria for the new "PE present" group, particularly the inclusion of the SSM.

Most importantly, communication with referring physicians, particularly in the Emergency Department, was much clearer and this was appreciated. Additionally, our Radiology and Nuclear Medicine residents interpreting the studies after hours expressed much greater comfort using the trinary system.

\section{Conclusion}

The radionuclide lung scan has traveled a long circuitous path since its introduction as a perfusion study in the mid1960s and a combined ventilation-perfusion study (V/Q) in 1969-70.

Its credibility has been questioned several times along the way, particularly after publication of the initially flawed PIOPED I study in 1990. However, many retrospective investigations on this early computer data-based study have given us great insight into how to significantly improve our interpretive skills.

The subsequent introduction of CTPA in the mid-1990s provided radiologists with a new means of investigating PE. Since diagnostic radiologists are inherently more comfortable interpreting anatomic rather than functional images, as a result, CTPA rapidly became the procedure of choice for investigating PE. Subsequent realization of the unacceptably high patient radiation exposure associated with CTPA, particularly to the female breast, has caused considerable concern to all. This, together with the everincreasing realization that we are overdiagnosing and 
overtreating PE with CTPA as well as with our improved diagnostic skills and the considerably lower radiation dose associated with $\mathrm{V} / \mathrm{Q}$, has re-awakened interest in this nuclear medicine procedure.

At the present time, it is clear that the V/Q scan should be the initial investigative tool in patients with a normal or close to normal chest radiograph. Follow-up studies on planar V/Q studies have shown a FN rate comparable to both SPECT V/Q and CTPA with their inherently greater sensitivities. We therefore propose that the eight-view planar V/Q study should be the initial procedure performed in suspected PE. In adopting this approach, a great deal can be accomplished toward avoiding the currently existing practice of overdiagnosis and overtreatment. The results of an ongoing investigation withholding anticoagulant therapy in patients with sub-segmental PE will, hopefully, finally convince clinicians that these small, frequently encountered PEs do not require treatment.

Conflict of interest Drs. Raphaella da Silva, Muhammad Shah and Leonard M. Freeman do not have any conflicts of interest.

Human and animal studies The retrospective data review in Table I was performed with the permission of our Institutional Review Board.

\section{References}

1. Remy-Jardin M, Remy J, Wattinne L et al (1992) Central pulmonary thromboembolism: diagnosis with spiral volumetric CT with the single-breath-hold technique-comparison with pulmonary angiography. Radiology 185:381-397

2. The PIOPED Investigators (1990) Value of the ventilation/perfusion scan in acute pulmonary embolism. Results of the prospective investigation of pulmonary embolism diagnosis. (PIOPED). JAMA 263:2753-2759

3. Biello DR, Mattar AJ, McKnight RC et al (1979) Ventilation/ perfusion studies in suspected pulmonary embolism. Am J Roentegenol 133:1033-1037

4. Miniati M, Pistolesi M, Marini C et al (1996) Value of perfusion lung scan in the diagnosis of pulmonary embolism: results of the prospective investigative study of acute pulmonary embolism (PISA-PED). Am J Res Crit Care Med 154:387-393

5. McNeil BJ (1980) Ventilation-perfusion studies and the diagnosis of pulmonary embolism:concise communication. J Nucl Med 21:319-323

6. Stein PD, Fowler SE, Goodman LR et al (2006) Multi-detector computed tomography for acute pulmonary embolism. N Engl J Med 354:2317-2327

7. Gottschalk A, Stein PD, Sostman HD et al (2007) Very low probability interpretation of V/Q lung scan in combination with low probability objective clinical assessment reliably excludes pulmonary embolism. Data from PIOPED II. J Nucl Med 48:1411-1415

8. Gottschalk A, Sostman HD, Coleman RE et al (1993) Ventilation perfusion scintigraphy in the PIOPED study. Part II. Evaluation of the scintigraphic criteria and interpretation. J Nucl Med 34:119-126

9. Freeman LM, Krynyckyi B, Zuckier LS (2001) Enhanced lung scan diagnosis of pulmonary embolism with the use of ancillary scintigraphic findings and clinical correlation. Sem Nucl Med 31:143-157

10. Stein PD, Henry JW, Gottschalk A (1993) The addition of clinical assessment to stratification according to prior cardiopulmonary disease further optimizes the interpretation of ventilation perfusion lung scans in pulmonary embolism. Chest 104:1472-1476

11. Stein PD, Gottshalk A, Henry JA, Shivkumar K (1993) Stratification of patients according to prior cardiopulmonary disease and probability assessment based on the number of mismatched segmental equivalents. Chest 104:1461-1467

12. Sostman HD, Gottschalk A (1982) The stripe sign: a new sign for nondiagnosis of nonembolic defects on pulmonary perfusion scintigraphy. Radiology 142:737-741

13. Worsley DF, Kim CK, Alavi A et al (1993) Detailed analysis of patients with matched ventilation-perfusion defects and chest radiographic opacities. J Nucl Med 34:1851-1853

14. Goldhaber S (2005) How chest CT for the diagnosis of pulmonary embolism has changed my professional life: reflections from a PE doctor. Sem Roentgenol 40:8-10

15. Fesmine FM, Brown MD, Espinosa JA (2011) Critical issues in the evaluation and management of adult patients presenting to the emergency department with suspected pulmonary embolism. Ann Emerg Med 57:628-653

16. Sostman HD, Gottschalk A (1992) Prospective validation of the stripe sign in ventilation- perfusion scintigraphy. Radiology 184:455-459

17. James AE, Conway JJ, Chang CH et al (1971) The fissure sign: its multiple causes. Am J Roentegenol Radium Ther Nucl Med 111:492-500

18. Gottschalk A, Stein P (1991) Lung scan perfusion defects limited to matching pleural effusions indicate a low probability of pulmonary embolism but do not confidently exclude the diagnosis. J Nucl Med 32:951 (Abstr.)

19. Caravallo P, Lavender JP (1989) The incidence and etiology of the ventilation/perfusion reverse mismatch. Clin Nucl Med 14:571-576

20. Sostman HD, Brown M, Toote A et al (1981) Perfusion scan in pulmonary vascular/lymphangitic carcinomatosis: the segmental contour pattern. Am J Roentegenol 137:1072-1074

21. Strauss EB, Sostman HD, Gottschalk A (1987) Radiographic parenchymal opacity, matching perfusion defect and normal ventilation. A sign of pulmonary embolus? Radiology 163:505-506

22. Chandra S, Sarkar PK, Chandra D (2013) Finding an alternative diagnosis does not justify increased use of CT-pulmonary angiography. BMC Pulm Med 13:9

23. Mamlouk MD, VanSonnenberg E, Gosalia R et al (2010) Pulmonary embolism at CT angiography: implications for appropriateness, cost and radiation exposure in 2003 patients. Radiology 256:625-632

24. Stein PD, Kayalh F, Olson RE (2004) Trends in the use of diagnostic imaging in patients hospitalized with acute pulmonary embolism. Am J Cardiol 93:1316-1317

25. Brenner DJ, Hall EJ (2007) Computed tomography: an increasing source of radiation exposure. N Engl J Med 357:2272-2284

26. Einstein AL, Henzlova MJ, Rajagopolan S (2007) Estimating risk of cancer associated with radiation exposure from 64 slice computed tomography coronary angiography. JAMA 298:317-323

27. Task Group on Control of Radiation Dose in Computed Tomography (2000) Managing patient dose in computed tomography. A report of the international commission on radiologic protection. Ann ICRP 30:7-45

28. Redberg RF, Smith-Bindman R (2014) We are giving ourselves cancer. NY times Op-Ed section, January 31, 2014

29. Pahade JF, Litmanovich D, Pedrosa I et al (2009) Imaging pregnant patients with suspected pulmonary embolism: what the radiologist needs to know. Radiographics 29:639-654 
30. Parker MS, Hui FK, Camacho MA (2005) Female breast radiation exposure during CT pulmonary angiography. Am J Roentgenol 185:1228-1231

31. Diederich S, Lenzen H (2000) Radiation exposure associated with imaging of the chest: comparison of different radiographic and computed tomography techniques. Cancer 89(Suppl. 11):2457-2460

32. McCullough CH, Liu HH (1995) Breast dose during electron beam CT: measurement with film dosimetry. Radiology 196:153-157

33. Leung AN, Bull TM, Jaeschke $\mathrm{R}$ et al (2011) An official American thoracic society/of thoracic radiology clinical practice guidelines: evaluation of suspected pulmonary embolism in pregnancy. Am J Resp Crit Care Med 184:1200-1208

34. Stein EG, Haramati LB, Chamarthy $M$ et al (2010) Success of a safe and simple algorithm to reduced use of CT pulmonary angiography in the emergency department. Am J Roentgenol 194:392-397

35. Anderson DR, Kahn SR, Rodger MA et al (2007) Computed tomographic pulmonary angiography vs. ventilation/perfusion lung scanning in patients with suspected pulmonary embolism. JAMA 298:2743-2753

36. Goodman L (2005) Small pulmonary emboli. What do we know? Radiology 234:654-658

37. Weiner RS, Schwartz L, Woloshin S (2013) When a test is too good: how CT pulmonary angiograms find pulmonary emboli that do not need to be found. BMJ 347:f3368

38. Stein PD, Henry JW (1997) Prevalence of acute pulmonary embolism in central and sub segmental pulmonary arteries and relation to probability interpretation of ventilation/perfusion lung scans. Chest 111:1246-1248

39. Minet C, Lugosi M, Savoye PV et al (2012) Pulmonary embolism in mechanically ventilated patients requiring computed tomography: prevalence, risk factors and outcome. Crit Care Med 40:3202-3208

40. Ritchie G, McGurks J, McCreath C et al (2007) Prospective evaluation of unsuspected pulmonary embolism on contrast enhanced multidetector CT (MDCT) scanning. Thorax 62:536-540

41. Schultz DJ, Brasel KJ, Washington L et al (2004) Incidence of asymptomatic pulmonary embolism in moderately to severely injured trauma patients. J Trauma 56:727-731 discussion 731-3

42. Robin ED (1977) Over diagnosis and overtreatment of pulmonary embolism: the emperor may have no clothes. Ann Intern Med $87: 775-781$
43. Burge AJ, Freeman KD, Klapper PI, Haramati LB (2008) Increased diagnosis of pulmonary embolism without a corresponding decline in mortality during the $\mathrm{CT}$ era. Clin Radiol 63:381-386

44. Sheh SH, Bellin E, Freeman KD, Haramati LB (2012) Pulmonary embolism diagnosis and mortality with CT pulmonary angiography versus ventilation perfusion scintigraphy: evidence of over diagnosis with CT? AJR Am J Roentgenol 198:1340-1345

45. Donato AA, Khoche S, Santora J, Wagner B (2010) Clinical outcomes in patients with isolated sub segmental pulmonary emboli diagnosed by multi-detector CT pulmonary angiography. Thromb Res 126:266-270

46. Stein PD, Goodman LR, Hull RD et al (2012) Diagnosis and management of isolated subsegmental pulmonary embolism: review and assessment of the options. Clin Appl Thromb Hemost $18: 20-26$

47. Clinical Trials. Gov, Identifier NCT 10455818

48. Freeman LM, Blaufox MD (2010) SPECT V/Q imaging of the lungs. Semin Nucl Med 40:393-479

49. Bajc M, Maffioli L, Miniati M (2014) Good clinical practice in pulmonary embolism diagnosis: where do we stand today? Eur J Nucl Med Mol Imaging 41:333-336

50. Reinartz P, Wildberger JE, Schoefer W et al (2004) Tomographic imaging in the diagnosis of pulmonary embolism: a comparison between V/Q lung scintigraphy in SPECT technique and multislice spiral CT. J Nucl Med 45:1501-1508

51. Bajc M, Neilly JB, Miniati M et al (2009) EANM guidelines for ventilation/perfusion scintigraphy. Eur J Nucl Med Mol Imaging 36:1356-1370

52. Leblanc M, Leveille F, Turcotte E (2007) Prospective evaluation of the negative predictive value of V/Q SPECT using ${ }^{99 \mathrm{~m}} \mathrm{Tc}$ Technegas. Nucl Med Commun 28:667-672

53. He J, Wang F, Dai H-J et al (2012) Chinese multi-center study of lung scintigraphy and CT pulmonary angiography for the diagnosis of pulmonary embolism. Int $\mathbf{J}$ Cardiovasc Imaging 28:1799-1805

54. Gray HW, McKillop JH, Berensen RG (1993) Lung scan reports: interpretation by clinicians. Nucl Med Commun 14:989-994

55. Gray HW, McKillop JH, Berensen RG (1993) Lung scan reporting language: what does it mean? Nucl Med Commun 14:1084-1087

56. Glaser JE, Chamarthy M, Haramati L et al (2011) Successful and safe implementation of a trinary interpretation and reporting strategy for V/Q lung scintigraphy. J Nucl Med 52:1-5 